\title{
Reporting of a surveillance system of multidrug- resistant organisms in an Italian hospital
}

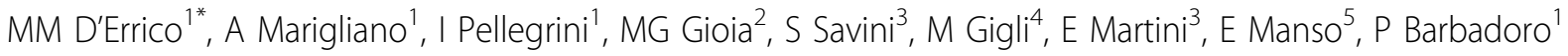 \\ From International Conference on Prevention \& Infection Control (ICPIC 2011) \\ Geneva, Switzerland. 29 June - 2 July 2011
}

\section{Introduction / objectives}

Healthcare facilities are monitoring Multidrug-Resistant Organisms (MDROs) because of their increasing incidence. A surveillance system for MDROs isolated from routine clinical cultures was set up in a teaching hospital in Central Italy.

\section{Methods}

Since January 2009, daily, Hospital Hygiene Service personnel collects MDROs microbiological data. Data are automatically entered in a software, continuously updated.Only the first MDRO isolate recovered from a patient is considered. The monthly report includes: newly recovered MDRO isolates; incidence rate of MDRO infection/colonization (No. of first MDRO isolates per patient for each unit/1000 patient days);statistical process control charts.

\section{Results}

From $01 / 01 / 2009$ to $31 / 12 / 2010,1160$ MDROs were isolated; the most represented MDRO was ESBL E.coli $(\mathrm{n}=216 ; 18.6 \%)$ followed by MRSA $(\mathrm{n}=178 ; 15.3 \%)$ and $A$. baumannii $(\mathrm{n}=158 ; 13.6 \%) .26 \%(\mathrm{n}=302)$ of the MDROs isolated came from Intensive Care Units (ICUs). The mean MDROs incidence rate was 1.96 and $10.68 / 1000$ patient days when considering the whole hospital and the ICUs, respectively. Analyzing the rates over time, an extremely fluctuating trend was observed: the ICUs MDROs rate varied from a minimum of 4.73 to a maximum of 20.88. No outbreaks were documented and the warning and control limits were never been exceeded. Among the possible factors contributing to this phenomenon, the sampling rate (No. of all samples sent to the microbiology laboratory/patient days) was analyzed, but no significant changes over time were found.

${ }^{1}$ Polytechnic University of The Marches, Ancona, Italy

Full list of author information is available at the end of the article

\section{Conclusion}

Our data highlighted the changeable trend of MDROs rates; a more accurate study is ongoing to assess what kind of variables can influence the spread of MDROs (staff? mini-clusters?) in order to provide the best preventive strategies.

\section{Disclosure of interest}

None declared.

\section{Author details}

${ }^{1}$ Polytechnic University of The Marches, Ancona, Italy. ${ }^{2}$ Hospital Hygiene Service, AOU Ospedali Riuniti, Ancona, Italy. ${ }^{3}$ AOU Ospedali Riuniti, Hospital Hygiene Service, Ancona, Italy. ${ }^{4}$ Hospital Hygiene Service, Ancona, Italy. ${ }^{5}$ Microbiology Laboratory, AOU Ospedali Riuniti, Ancona, Italy.

Published: 29 June 2011

doi:10.1186/1753-6561-5-S6-P298

Cite this article as: D'Errico et al.: Reporting of a surveillance system of multidrug-resistant organisms in an Italian hospital. BMC Proceedings 2011 5(Suppl 6):P298.

Submit your next manuscript to BioMed Central and take full advantage of:

- Convenient online submission

- Thorough peer review

- No space constraints or color figure charges

- Immediate publication on acceptance

- Inclusion in PubMed, CAS, Scopus and Google Scholar

- Research which is freely available for redistribution 